\title{
ON THE ESTIMATED VARIANCES OF REGRESSION COEFFICIENTS IN MISSPECIFIED ERROR COMPONENTS MODELS
}

\author{
PhiLIPPE J. Deschamps \\ University of Fribourg
}

\begin{abstract}
In a regression model with an arbitrary number of error components, the covariance matrix of the disturbances has three equivalent representations as linear combinations of matrices. Furthermore, this property is invariant with respect to powers, matrix addition, and matrix multiplication. This result is applied to the derivation and interpretation of the inconsistency of the estimated coefficient variances when the error components structure is improperly restricted. This inconsistency is defined as the difference between the asymptotic variance obtained when the restricted model is correctly specified, and the asymptotic variance obtained when the restricted model is incorrectly specified; when some error components are improperly omitted, and the remaining variance components are consistently estimated, it is always negative. In the case where the time component is improperly omitted from the two-way model, we show that the difference between the true and estimated coefficient variances is of order greater than $N^{-1}$ in probability.
\end{abstract}

\section{INTRODUCTION}

Since the pioneering article by Balestra and Nerlove [3], many authors have studied the estimation of linear regression models with error components. The Balestra-Nerlove specification was limited to a two-way classification of data, and omitted time-specific effects. This restriction was later lifted by Wallace and Hussain [10] and Nerlove [8], who considered the following error specification:

$u_{i t}=v_{i}+w_{t}+\epsilon_{i t} \quad$ for $i=1, \ldots, n$ and $t=1, \ldots, T$

where $v_{i}, w_{t}$, and $\epsilon_{i t}$ have zero expectations, constant variances, and are pairwise and serially uncorrelated. Nerlove, in particular, found the spectral form of the error covariance matrix $V$ implied by equation (1), expressing $V$ as a linear combination of symmetric, idempotent, and pairwise orthog-

Part of this research was conducted while the author held a Faculty Research Fellowship at CentER, Tilburg University. I wish to thank Pietro Balestra, Badi Baltagi, and anonymous referees for helpful comments. A previous version of this paper was presented at CORE, CentER, and at the European Meeting of the Econometric Society, Munich, 1989. Any remaining error is my own. 
onal matrices that sum to an identity matrix. All the rational powers of $V$ can be similarly represented; this is particularly useful for interpreting and computing the Aitken estimators of the regression coefficients. The spectral form also leads to a particularly simple expression of the ANOVA table for the variance components of $u_{i t}$ (for a classical presentation of this table, see, e.g., Graybill [6, p. 349]).

More recently, Searle and Henderson [9] and Deschamps [4] have studied the structure of the error covariance matrix in a model with a general, $p$-way classification of data (where there are $2^{p}-1$ error components). As will be shown, their results imply that $V$ has three equivalent representations as linear combinations of matrices. The coefficients of the first one are the variance components; the coefficients of the second one (the spectral form) are the eigenvalues of $V$; the coefficients of the third one are the elements of $V$. Each linear combination is, in a sense, isomorphic to the two others. Furthermore, the property is invariant with respect to powers, linear combinations, and matrix multiplication. This means that if the nonsingular matrices $V_{1}, V_{2}, V_{3}, V_{4}$ have the same form as $V$ and if $a, b, \alpha, \beta, \gamma, \delta$ are scalars, then $V_{1}^{\alpha}\left(a V_{2}^{\beta}+b V_{3}^{\gamma}\right) V_{4}^{\delta}$ also has the same form; it can be represented as three linear combinations of the same matrices that appear in the three expressions for $V$. Furthermore, the three sets of coefficients are easily derived.

Section 2 of this paper will present the assumptions of the model, and a new notation. In Section 3, we present Theorem 1, which is a general result on the misspecified error components model. Theorem 1 can be used to derive and interpret the inconsistency ( $\left.\operatorname{plim} N\left(\hat{\sigma}_{i}^{2}-\sigma_{i}^{2}\right)\right)$ of the estimated variances of the regression coefficients when some error components are improperly omitted (even though their variances are nonzero), and the remaining variance components are consistently estimated. We will show that the true coefficient variances are always underestimated in the misspecified model, for any $p$-way classification and for any number of omitted components. More generally, we find that the inconsistency can be expressed as three linear combinations of quadratic forms, in the same way as there are three representations of $V$. This turns out to be very helpful for the interpretation of the inconsistency.

When a single error component is omitted in the misspecified model and when the remaining variance components are consistently estimated, our results also imply that the inconsistency is proportional to the variance of the omitted component. The coefficient of proportionality is a linear combination of sums of squares of partial sums (as in, e.g., $\alpha_{1} \Sigma_{t}\left(\sum_{i} Z_{i t}\right)^{2}+$ $\left.\alpha_{2} \sum_{i}\left(\Sigma_{t} Z_{i t}\right)^{2}\right)$; it can be estimated as a by-product of generalized least squares on the misspecified model. This greatly facilitates the sensitivity analysis of misspecification.

Theorem 1 also applies to other types of misspecification: the inconsistency of an estimated coefficient variance is a linear combination of the inconsistencies of the variance component estimates. 
In Section 4, we illustrate the previous results by analyzing the inconsistency when the time-specific component $w_{t}$ is improperly omitted from equation (1). This case is of particular interest since it corresponds to the earliest (Balestra-Nerlove) specification, and is therefore found in most empirical implementations. In this instance the inconsistency has a particularly simple, intuitively appealing form, whose properties are easily investigated. It will be shown that this inconsistency is unbounded, unless the matrix of regressors satisfies very restrictive assumptions.

Lemma 1 of Appendix A presents the three isomorphic representations of $V$, and can be viewed as a synthesis of Searle and Henderson [9] and Deschamps [4]. This lemma will be used in Appendix B, where we prove Theorem 1 of Section 3. Appendix $C$ presents estimators of the variance components which are consistent under misspecification, and coincide with the ones proposed by Amemiya [1] for a correctly specified three-component model.

\section{A GENERAL ERROR COMPONENTS MODEL}

Our regression model has $2^{p}$ error components, one of which is identically zero. It can be written as

$y=X \beta+u$

where $y$ is an $N \times 1$ vector of observations on a dependent variable, $X$ is an $N \times k$ matrix of observations on nonstochastic regressors, $\beta$ is a $k \times 1$ vector of regression coefficients, and $u$ is an $N \times 1$ vector of compound disturbances with the following structure:

$u=\sum_{\alpha=00 \ldots 0}^{11 \ldots 1}\left(s_{n_{1}}^{1-\alpha_{1}} \otimes \cdots \otimes s_{n_{p}}^{1-\alpha_{p}}\right) v_{\alpha}$

with

$E\left(v_{\alpha}\right)=0_{q_{\alpha}}$

$E\left(v_{\alpha} v_{\alpha}^{\prime}\right)=\theta_{\alpha} I_{q_{\alpha}}$

$E\left(v_{\alpha} v_{\beta}^{\prime}\right)=O_{q_{\alpha} \times q_{\beta}} \quad(\alpha \neq \beta)$.

In equations (3a)-(3d) and in the rest of this paper, the Greek subscripts $\alpha, \beta$, and $\gamma$ indicate binary numbers with $p$ digits, for example, $\alpha=$ $\alpha_{1} \alpha_{2} \ldots \alpha_{p}$, where $\alpha_{i}$ is either zero or one. The letters $n_{1}, \ldots, n_{p}$ denote numbers of cells with $\prod_{i=1}^{p} n_{i}=N, s_{n_{i}}$ is an $n_{i} \times 1$ vector of ones, and we adopt the convention that $s_{n_{i}}^{0}=I_{n_{i}}$, an identity matrix of order $n_{i} . v_{\alpha}$ is the vector of the $q_{\alpha}=\Pi_{i=1}^{p} n_{i}^{\alpha_{i}}$ realizations of error component $\alpha$. This vector is assumed to have zero expectation and scalar covariance matrix in ( $3 \mathrm{~b}$ ) and (3c); equation (3d) assumes that the error components are pairwise uncorrelated. We take the first error component to be identically zero, and this implies that $\theta_{00 \ldots 0}=0$. 
Specification (3a)-(3d) obviously implies the following form for the covariance matrix of $u$ (see Note 1):

$$
V=E\left(u u^{\prime}\right)=\sum_{\alpha=00 \ldots 0}^{11 \ldots 1} \theta_{\alpha}\left(S_{n_{1}}^{1-\alpha_{1}} \otimes \cdots \otimes S_{n_{p}}^{1-\alpha_{p}}\right)
$$

where $S_{n_{i}}$ is a square matrix of ones of order $n_{i}$ and $S_{n_{i}}^{0} \equiv I_{n_{i}}$.

The model in Nerlove [8] is obtained as a special case of (4) by letting $p=2, n_{1}=n$, and $n_{2}=T$ :

$$
V=\theta_{00} S_{n T}+\theta_{01}\left(S_{n} \otimes I_{T}\right)+\theta_{10}\left(I_{n} \otimes S_{T}\right)+\theta_{11} I_{n T}
$$

where $\theta_{00}=0, \theta_{01} \equiv \sigma_{w}^{2}, \theta_{10} \equiv \sigma_{v}^{2}$, and $\theta_{11} \equiv \sigma_{\epsilon}^{2}$ (compare equation (1)). By letting $\theta_{01}=0$ in (5), the model specializes further to Balestra and Nerlove [3].

The reader will have no difficulty in verifying that when $p=3$, if $\theta_{000}=$ $\theta_{001}=\theta_{010}=\theta_{011}=\theta_{101}=0$ and if $\theta_{100} \equiv \sigma_{v}^{2}, \theta_{110} \equiv \sigma_{w}^{2}, \theta_{111} \equiv \sigma_{\epsilon}^{2}$, equation (4) specializes to the nested specification in Fuller and Battese [5], implied by

$u_{i j t}=v_{i}+w_{i j}+\epsilon_{i j t}$.

So the binary number $\alpha$ may serve to interpret the variance component $\theta_{\alpha}$ in equation (4): it is simply the variance of that error term which has the particular index subset identified by the unitary digits in $\alpha$.

Equation (4) is the first of our three isomorphic characterizations of $V$; the two others are given in Appendix A.

\section{THE CONSEQUENCES OF MISSPECIFICATION}

We consider the case where $V$ in equation (4) is estimated by:

$$
\hat{V}_{*}=\sum_{\alpha=00 \ldots 0}^{11 \ldots 1} \hat{\theta}_{\alpha}^{*}\left(\bigotimes_{i=1}^{p} S_{n_{i}}^{1-\alpha_{i}}\right)
$$

where $\hat{\theta}^{*}$ is a possibly inconsistent estimator of the $2^{p} \times 1$ vector $\theta$. We estimate $\beta$ by

$\hat{\beta}=\left(X^{\prime} \hat{V}_{*}^{-1} X\right)^{-1} X^{\prime} \hat{V}_{*}^{-1} y$.

The covariance matrix of $\hat{\beta}$ is estimated by:

$C=\left(X^{\prime} \hat{V}_{*}^{-1} X\right)^{-1}$

whereas the true covariance matrix, conditional on $\hat{\theta}^{*}$, is $V\left(\hat{\beta} \mid \hat{\theta}^{*}\right)=\left(X^{\prime} \hat{V}_{*}^{-1} X\right)^{-1} X^{\prime} \hat{V}_{*}^{-1} V \hat{V}_{*}^{-1} X\left(X^{\prime} \hat{V}_{*}^{-1} X\right)^{-1}$.

We let $\theta^{*}=\operatorname{plim} \hat{\theta}^{*}$ (see Note 2), and assume that $V_{*}=\sum_{\alpha} \theta_{\alpha}^{*} \bigotimes_{i=1}^{p} S_{n_{i}}^{1-\alpha_{i}}$ is positive definite. We also assume that $X^{\prime} V_{*}^{-1} X / N$ tends to a finite, positive definite matrix $M_{*}$. We further note that

$N C=\left(\frac{X^{\prime} \hat{V}_{*}^{-1} X}{N}\right)^{-1}$ 
and

$N V\left(\hat{\beta} \mid \hat{\theta}^{*}\right)=\left(\frac{X^{\prime} \hat{V}_{*}^{-1} X}{N}\right)^{-1}\left(\frac{X^{\prime} \hat{V}_{*}^{-1} V \hat{V}_{*}^{-1} X}{N}\right)\left(\frac{X^{\prime} \hat{V}_{*}^{-1} X}{N}\right)^{-1}$

We wish to evaluate

$\operatorname{plim} N\left(C-V\left(\hat{\beta} \mid \hat{\theta}^{*}\right)\right)$

$$
\begin{aligned}
& =\operatorname{plim} N\left(\left(X^{\prime} \hat{V}_{*}^{-1} X\right)^{-1}-\left(X^{\prime} \hat{V}_{*}^{-1} X\right)^{-1} X^{\prime} \hat{V}_{*}^{-1} V \hat{V}_{*}^{-1} X\left(X^{\prime} \hat{V}_{*}^{-1} X\right)^{-1}\right) \\
& =\lim N\left(\left(X^{\prime} V_{*}^{-1} X\right)^{-1}-\left(X^{\prime} V_{*}^{-1} X\right)^{-1} X^{\prime} V_{*}^{-1} V V_{*}^{-1} X\left(X^{\prime} V_{*}^{-1} X\right)^{-1}\right) \\
& =\lim N\left(\left(X^{\prime} V_{*}^{-1} X\right)^{-1}\left(X^{\prime} V_{*}^{-1} V_{*} V_{*}^{-1} X-X^{\prime} V_{*}^{-1} V V_{*}^{-1} X\right)\left(X^{\prime} V_{*}^{-1} X\right)^{-1}\right) \\
& =M_{*}^{-1} \lim \left(\frac{X_{*}^{\prime} D X_{*}}{N}\right) M_{*}^{-1}
\end{aligned}
$$

with $X_{*}=V_{*}^{-1 / 2} X$ and $D=V_{*}^{-1 / 2}\left(V_{*}-V\right) V_{*}^{-1 / 2}$.

Equation (9) implies the following expression for the inconsistency of the estimated variance of $\sqrt{N} \hat{\beta}_{p}$ :

$\operatorname{plim} N\left(C_{\ell^{\prime}}-V\left(\hat{\beta}_{\prime^{\prime}} \mid \hat{\theta}^{*}\right)\right)=\lim \left(\frac{Z^{\prime}(\ell) D Z(\ell)}{N}\right)$

where $Z(\ell)$ is the $\ell$ th column of the matrix $X_{*} M_{*}^{-1}$.

The following definition provides the key to the three equivalent representations of $V$ given in Appendix $\mathrm{A}$, and to the three corresponding representations of (10) given by Theorem 1 of this section.

DEFINITION 1. Let, as before, $S_{n_{i}}$ be a matrix of ones of order $n_{i}$ and zero powers denote identity matrices. We define

$$
\begin{aligned}
& K=\bigotimes_{i=1}^{p}\left(\begin{array}{ll}
1 & 0 \\
1 & 1
\end{array}\right) ; \\
& K_{0}=\bigotimes_{i=1}^{p}\left(\begin{array}{ll}
n_{i} & 1 \\
0 & 1
\end{array}\right) ; \\
& L_{\beta}=\bigotimes_{i=1}^{p} S_{n_{i}}^{1-\beta_{i}} ; \\
& R_{\beta}=\bigotimes_{i=1}^{p}\left(\frac{1}{n_{i}} S_{n_{i}}\right)^{1-\beta_{i}}\left(I_{n_{i}}-\frac{1}{n_{i}} S_{n_{i}}\right)^{\beta_{i}} ; \\
& M_{\beta}=\bigotimes_{i=1}^{p}\left(S_{n_{i}}-I_{n_{i}}\right)^{1-\beta_{i}} .
\end{aligned}
$$

We now define $\eta=\theta^{*}-\theta$ as the vector of variance component inconsistencies. It is shown in Appendix $\mathrm{A}$ that the elements of $K_{0} \theta^{*}$ are the eigenvalues of $V_{*}$, so that they are strictly positive by assumption. Theorem 1 shows that the inconsistency (10) of an estimated coefficient variance is a 
linear combination of the elements of $\eta$, with coefficients that depend on $\theta^{*}$, but not on $\theta$.

THEOREM 1. Let $\eta=\operatorname{plim} \hat{\theta}^{*}-\theta=\theta^{*}-\theta$, let $V_{*}=\Sigma_{\alpha} \theta_{\alpha}^{*} L_{\alpha}$, let $D=$ $V_{*}^{-1 / 2}\left(V_{*}-V\right) V_{*}^{-1 / 2}$, and let $\Delta$ be the diagonal matrix with the elements of $K_{0} \theta^{*}$ on the diagonal. Then $D$ can be equivalently expressed as

$$
\begin{aligned}
& D=\sum_{\gamma=00 \ldots 0}^{11 \ldots 1} \eta_{\gamma} \sum_{\beta=00 \ldots 0}^{11 \ldots 1} a_{\beta \gamma} R_{\beta} \\
& D=\sum_{\gamma=00 \ldots 0}^{11 \ldots 1} \eta_{\gamma} \sum_{\beta=00 \ldots 0}^{11 \ldots 1} b_{\beta \gamma} L_{\beta} \\
& D=\sum_{\gamma=00 \ldots 0}^{11 \ldots 1} \eta_{\gamma} \sum_{\beta=00 \ldots 0}^{11 \ldots 1} c_{\beta \gamma} M_{\beta}
\end{aligned}
$$

where $a_{\beta \gamma}, b_{\beta \gamma}$, and $c_{\beta \gamma}$ are the elements in row $\beta$ and column $\gamma$ of $\Delta^{-1} K_{0}$, $K_{0}^{-1} \Delta^{-1} K_{0}$, and $K K_{0}^{-1} \Delta^{-1} K_{0}$, respectively.

The most interesting type of misspecification occurs when one, or several, variance components $\theta_{\gamma}$ are improperly excluded from equation (4). This follows from letting $\hat{\theta}_{\gamma}^{*}=0$, so that $\theta_{\gamma}^{*}=0$. It is shown in Appendix $C$ that the remaining variance components can always be consistently estimated. In this case the inconsistency (10) is always negative: indeed, it is shown in Appendix A that the matrices $R_{\beta}$ in (16) are idempotent, pairwise orthogonal, and add up to an identity matrix, so that the eigenvalues of $D$ are $\lambda_{\beta}=$ $\sum_{\gamma} \eta_{\gamma} a_{\beta \gamma}$. Since $a_{\beta \gamma} \geq 0$ and since $\eta_{\gamma}=0$ when component $\gamma$ is included, whereas $\eta_{\gamma}=-\theta_{\gamma} \leq 0$ when component $\gamma$ is excluded, we have that $\lambda_{\beta} \leq 0$ for all $\beta$.

We may also note that the opposite type of misspecification whereby a variance component $\theta_{\gamma}$ is improperly included is of no consequence asymptotically if plim $\hat{\theta}_{\gamma}^{*}=\theta_{\gamma}=0$, so that $\eta_{\gamma}=0$ in Theorem 1 .

Equation (17) implies that the quadratic form in (10) is a linear combination of sums of squares of partial sums of the elements of $Z(\ell)$, as is obvious from the definition of $L_{\beta}$ in (13). This fact will be used in Section 4. Similarly, equation (18) expresses the quadratic form as a linear combination of sums of cross-products of the elements of $Z(\ell)$ (see the definition of $M_{\beta}$ in (15)). Using the arguments in Appendix A, it is easy to show that the coefficients $\Sigma_{\gamma} \eta_{\gamma} c_{\beta \gamma}$ in equation (18) are in fact the elements of $D$.

\section{AN ILLUSTRATION}

In this section, we illustrate the preceding theorem by considering the case where $w_{t}$ is improperly omitted from equation (1); equivalently, $\hat{\theta}_{01}^{*}=0$ in equation (7). We will first consider the case where the two remaining variance components are consistently estimated (Appendix $\mathrm{C}$ shows that this will be true if the two-way formulas are used). This assumption will be relaxed 
at the end of this section. For now, we assume that $\theta_{00}^{*}=\theta_{01}^{*}=0, \theta_{10}^{*}=\theta_{10}$, and $\theta_{11}^{*}=\theta_{11}$.

As previously noted, the elements of $K_{0} \theta^{*}$ in Theorem 1 are the eigenvalues of $V_{*}$. Using equation (12), they may be written as

$$
\left(\begin{array}{c}
\lambda_{00} \\
\lambda_{01} \\
\lambda_{10} \\
\lambda_{11}
\end{array}\right)=\left(\begin{array}{cccc}
n T & n & T & 1 \\
0 & n & 0 & 1 \\
0 & 0 & T & 1 \\
0 & 0 & 0 & 1
\end{array}\right)\left(\begin{array}{c}
0 \\
0 \\
\theta_{10} \\
\theta_{11}
\end{array}\right)=\left(\begin{array}{c}
T \theta_{10}+\theta_{11} \\
\theta_{11} \\
T \theta_{10}+\theta_{11} \\
\theta_{11}
\end{array}\right) .
$$

Furthermore, the coefficients $b_{\beta \gamma}$ in equation (17) are elements of the matrix:

$$
\begin{aligned}
& K_{0}^{-1} \Delta^{-1} K_{0} \\
& =\frac{1}{n T}\left(\begin{array}{cccc}
1 & -1 & -1 & 1 \\
0 & T & 0 & -T \\
0 & 0 & n & -n \\
0 & 0 & 0 & n T
\end{array}\right)\left(\begin{array}{cccc}
\lambda_{00}^{-1} & 0 & 0 & 0 \\
0 & \lambda_{01}^{-1} & 0 & 0 \\
0 & 0 & \lambda_{10}^{-1} & 0 \\
0 & 0 & 0 & \lambda_{11}^{-1}
\end{array}\right)\left(\begin{array}{cccc}
n T & n & T & 1 \\
0 & n & 0 & 1 \\
0 & 0 & T & 1 \\
0 & 0 & 0 & 1
\end{array}\right) \\
& =\frac{1}{n T}\left(\begin{array}{cccc}
n T \lambda_{00}^{-1} & n\left(\lambda_{00}^{-1}-\lambda_{01}^{-1}\right) & T\left(\lambda_{00}^{-1}-\lambda_{10}^{-1}\right) & \lambda_{00}^{-1}-\lambda_{01}^{-1}-\lambda_{10}^{-1}+\lambda_{11}^{-1} \\
0 & n T \lambda_{01}^{-1} & 0 & T\left(\lambda_{01}^{-1}-\lambda_{11}^{-1}\right) \\
0 & 0 & n T \lambda_{10}^{-1} & n\left(\lambda_{10}^{-1}-\lambda_{11}^{-1}\right) \\
0 & 0 & 0 & n T \lambda_{11}^{-1}
\end{array}\right] .
\end{aligned}
$$

Since the only inconsistency occurs in the time component, we have $\eta_{01}=$ $-\theta_{01}$ and $\eta_{00}=\eta_{10}=\eta_{11}=0$, so that we only need those coefficients $b_{\beta \gamma}$ that are located in the second column of the preceding matrix (which has the binary index 01). Upon substituting (13), (19), and (20) in equation (17), we obtain

$$
\begin{aligned}
D & =-\theta_{01}\left(\frac{1}{T}\left(\frac{1}{T \theta_{10}+\theta_{11}}-\frac{1}{\theta_{11}}\right) S_{n T}+\frac{1}{\theta_{11}}\left(S_{n} \otimes I_{T}\right)\right) \\
& =-\frac{\theta_{01}}{\theta_{11}}\left(\frac{-\theta_{10}}{T \theta_{10}+\theta_{11}} S_{n T}+\left(S_{n} \otimes I_{T}\right)\right)
\end{aligned}
$$

so that the quadratic form in equation $(10)$ is

$$
Z^{\prime}(\ell) D Z(\ell)=-\frac{\theta_{01}}{\theta_{11}}\left(\sum_{t=1}^{T} Z_{. t}^{2}(\ell)-\frac{\theta_{10}}{T \theta_{10}+\theta_{11}} Z_{. .}^{2}(\ell)\right)
$$

with $Z_{. t}(\ell)=\sum_{i=1}^{n} Z_{i r}(\ell)$ and $Z_{. .}(\ell)=\sum_{i=1}^{n} \sum_{t=1}^{T} Z_{i t}(\ell)$. 
Since $\theta_{10} /\left(T \theta_{10}+\theta_{11}\right)<1 / T$, an upper bound for the expression in (22) is given by

$Z^{\prime}(\ell) D Z(\ell) \leq-\frac{\theta_{01}}{\theta_{11}} \sum_{t=1}^{T}\left(Z_{. t}(\ell)-\frac{Z_{. .}(\ell)}{T}\right)^{2}$

and it is seen that for given $\theta_{01}$ and $\theta_{11}, \lim _{T \rightarrow \infty} Z^{\prime}(\ell) D Z(\ell)=-\infty$, unless $\theta_{01}=0$ or unless $Z_{. t}(\ell)=\alpha$ for all $t$. In the latter case, equation (22) is easily shown to imply

$Z^{\prime}(\ell) D Z(\ell)=\frac{-T \alpha^{2} \theta_{01}}{T \theta_{10}+\theta_{11}}$

whose limit equals $-\alpha^{2} \theta_{01} / \theta_{10}$ as $T$ tends to infinity.

To summarize, the inconsistency of the $\ell$ th estimated variance in the misspecified model is unbounded as $n \rightarrow \infty$ and $T \rightarrow \infty$. This may be seen by dividing equation (22) by $n T$, and noting that $\sum_{t=1}^{T} Z_{. t}^{2}(\ell) / n T$ does not converge in general. The unboundedness occurs unless the "observations" in the $\ell$ th column of $X_{*} M_{*}^{-1}$ repeat their average pattern over time. In this (very special) case where $Z_{. t}(\ell)=\alpha$ for all $t$, we have

$\operatorname{plim} n T\left(C_{\rho \rho}-V\left(\hat{\beta}_{\rho} \mid \hat{\theta}^{*}\right)\right)=\lim \frac{Z^{\prime}(\ell) D Z(\ell)}{n T}=\lim \frac{1}{n T}\left(\frac{-\alpha^{2} \theta_{01}}{\theta_{10}}\right)=0$.

We will now show that the previous result remains essentially unchanged when $\hat{\theta}_{10}^{*}$ and $\hat{\theta}_{11}^{*}$ are the one-way estimators:

$\hat{\theta}_{11}^{*}=\frac{1}{n(T-1)}\left(\hat{u}^{\prime}\left(I_{n} \otimes R_{1}\right) \hat{u}\right)$

$\hat{\theta}_{10}^{*}=\frac{1}{T}\left(\frac{\hat{u}^{\prime}\left(I_{n} \otimes R_{0}\right) \hat{u}}{n}-\frac{\hat{u}^{\prime}\left(I_{n} \otimes R_{1}\right) \hat{u}}{n(T-1)}\right)$

where $R_{0}=T^{-1} S_{T}, R_{1}=I_{T}-R_{0}$, and where $\hat{u}$ is a predictor of $u$. If the true disturbances $u_{i t}$ are known and $\hat{u}=u$, it is easy to show that $E\left(\hat{\theta}_{11}^{*}\right)=$ $\theta_{01}+\theta_{11}$ and $E\left(\hat{\theta}_{10}^{*}\right)=\theta_{10}$; we will therefore assume that $\theta_{11}^{*}=\theta_{01}+\theta_{11}$ and that $\theta_{10}^{*}=\theta_{10}$. As before, we have $\theta_{00}^{*}=\theta_{01}^{*}=0$. The eigenvalues of $V_{*}$ are again given by $K_{0} \theta^{*}$, this time as

$\left(\begin{array}{c}\lambda_{00} \\ \lambda_{01} \\ \lambda_{10} \\ \lambda_{11}\end{array}\right)=\left(\begin{array}{c}T \theta_{10}+\theta_{01}+\theta_{11} \\ \theta_{01}+\theta_{11} \\ T \theta_{10}+\theta_{01}+\theta_{11} \\ \theta_{01}+\theta_{11}\end{array}\right)$. 
The vector $\eta$ of variance component inconsistencies is this time given by:

$$
\left(\begin{array}{c}
\eta_{00} \\
\eta_{01} \\
\eta_{10} \\
\eta_{11}
\end{array}\right)=\left(\begin{array}{c}
0 \\
-\theta_{01} \\
0 \\
\theta_{01}
\end{array}\right) .
$$

Upon using again the methodology of Theorem 1 , we see that $D=D_{1}+$ $D_{2}$, with

$$
\begin{aligned}
& D_{1}=-\theta_{01}\left(\frac{1}{T}\left(\frac{1}{T \theta_{10}+\theta_{01}+\theta_{11}}-\frac{1}{\theta_{01}+\theta_{11}}\right) S_{n T}+\frac{1}{\theta_{01}+\theta_{11}}\left(S_{n} \otimes I_{T}\right)\right) \\
& D_{2}=\theta_{01}\left(\frac{1}{T}\left(\frac{1}{T \theta_{10}+\theta_{01}+\theta_{11}}-\frac{1}{\theta_{01}+\theta_{11}}\right)\left(I_{n} \otimes S_{T}\right)+\frac{1}{\theta_{01}+\theta_{11}} I_{n T}\right) .
\end{aligned}
$$

The matrix $D_{1}$ has the same form as $D$ in (21), with $\theta_{11}$ replaced by $\theta_{01}+$ $\theta_{11}$; hence the preceding analysis also applies to $Z^{\prime}(\ell) D_{1} Z(\ell) / n T$, which is negative and generally unbounded. By analogy with (22), the second term may be written as

$$
\begin{aligned}
Z^{\prime}(\ell) D_{2} Z(\ell) & =\frac{\theta_{01}}{\theta_{01}+\theta_{11}}\left(\sum_{i=1}^{n} \sum_{t=1}^{T} Z_{i t}^{2}(\ell)-\frac{\theta_{10}}{T \theta_{10}+\theta_{01}+\theta_{11}} \sum_{i=1}^{n} Z_{i .}^{2}(\ell)\right) \\
& \geq \frac{\theta_{01}}{\theta_{01}+\theta_{11}} \sum_{i=1}^{n} \sum_{t=1}^{T}\left(Z_{i t}(\ell)-\frac{Z_{i .}(\ell)}{T}\right)^{2} \geq 0
\end{aligned}
$$

and $Z^{\prime}(\ell) D_{2} Z(\ell) / n T$ converges to a finite, nonnegative number if $\sum_{i, t} Z_{i t}^{2}(\ell) /$ $n T$ and $\Sigma_{i} Z_{i .}^{2}(\ell) / n T^{2}$ are bounded. Furthermore the lower bound in (23) vanishes if, and only if, $Z_{i t}(\ell)=c_{i}$ for all $t$; in this case the limit of $Z^{\prime}(\ell) D_{2} Z(\ell) / n T$ is easily shown to vanish when $\theta_{10} \neq 0$.

Collecting results, we may say that the inconsistency remains negative and generally unbounded, since the negative term dominates the positive one. Furthermore, when $\theta_{10} \neq 0$, the inconsistency now vanishes if (and only if) the elements of the relevant column of $X_{*} M_{*}^{-1}$ exactly repeat their pattern over time.

\section{CONCLUDING REMARKS}

In a general error components model with a $p$-way classification of data, we have given three interpretations of the inconsistency of the estimated coefficient variances when the error components structure is misspecified. In the special case where the time component is improperly omitted from the two- 
way classification model, this inconsistency is in general unbounded, in the sense that $C_{\rho \rho}-V\left(\hat{\beta}_{\ell} \mid \hat{\theta}^{*}\right)$ is of order greater than $N^{-1}$ in probability, so that plim $N V\left(\hat{\beta}_{p} \mid \hat{\theta}^{*}\right)$ does not exist. Since plim $N V\left(\hat{\beta}_{p} \mid \hat{\theta}^{*}\right)$ is the asymptotic variance when the restricted model is incorrectly specified, this implies that $\sqrt{N}\left(\hat{\beta}_{p}-\beta_{\ell}\right)$ does not have a proper limiting distribution in such a case.

The arguments in Appendixes $A$ and $C$ indicate that the $p$-way model is essentially a tensor generalization of the 2-way model, and is, as such, not much more difficult to handle. It is therefore unfortunate, in view of the potentially serious consequences of misspecification, that most contributions to the literature on error components have been limited to the BalestraNerlove specification.

\section{NOTES}

1. Note that equation (4) differs from Searle and Henderson [9, equation 2.2] in the indexing of the variance components $\theta_{\alpha}$. A correspondence between the two indexing schemes is obtained by writing the digits in $\alpha$ in reverse order, and taking the complement to unity of each digit: for instance, $\theta_{011}$ in our notation corresponds to $\theta_{001}$ in [9]. As we will see, our notation has definite advantages for interpreting the variance components, and for stating the isomorphisms of Appendix A.

2. Unless otherwise indicated, all the limits in this paper are taken as $n_{i} \rightarrow \infty$ for all $i$.

3. If $\hat{V}_{*}=\hat{\sigma}_{u}^{2} I$ with $\hat{\sigma}_{u}^{2}$ the OLS estimator of the error variance, it may be shown (Greenwald, [7]) that $\left(X^{\prime} X\right)^{-1} X^{\prime}\left(V_{*}-V\right) X\left(X^{\prime} X\right)^{-1}$ approximates the bias of the estimated covariance matrix when $N$ is large.

4. Note that $c_{\alpha \beta}=0$ for $\beta=00 \ldots 0$.

\section{REFERENCES}

1. Amemiya, $\mathrm{T}$. The estimation of the variances in a variance components model. International Economic Review 12 (1971): 1-13.

2. Balestra, P. Best quadratic unbiased estimators of the variance-covariance matrix in normal regression. Journal of Econometrics 1 (1973): 17-28.

3. Balestra, P. \& M. Nerlove. Pooling cross section and time series data in the estimation of a dynamic model: the demand for natural gas. Econometrica 34 (1966): 585-612.

4. Deschamps, P. A note on isomorphic characterizations of the dispersion matrix in error components models. Linear Algebra and Its Applications 111 (1988): 147-150.

5. Fuller, W.A. \& G.E. Battese. Transformations for estimation of linear models with nestederror structure. Journal of the American Statistical Association 68 (1973): 626-632.

6. Graybill, F.A. An Introduction to Linear Statistical Models, vol. I. New York: McGrawHill, 1961.

7. Greenwald, B.C. A general analysis of bias in the estimated standard errors of least squares coefficients. Journal of Econometrics 22 (1983): 323-338.

8. Nerlove, M. A note on error components models. Econometrica 39 (1971): 383-396.

9. Searle, S.R. \& H.V. Henderson. Dispersion matrices for variance components models. Journal of the American Statistical Association 74 (1979): 465-470.

10. Wallace, T.D. \& A. Hussain. The use of error components models in combining cross section with time series data. Econometrica 37 (1969): 55-72. 


\section{APPENDIX A. CHARACTERIZATIONS OF THE ERROR COVARIANCE MATRIX}

It is easily shown that the following lemma can be extended to all the rational powers of $V$, and also to linear combinations or products of matrices having the same structure as $V$ (an example of such an extension is in fact given by Theorem 1).

LEMMA 1. Let $u$ have the specification $(3 a)-(3 d)$, and let $V=E\left(u u^{\prime}\right)$. Then $V=\sum_{\alpha=00 \ldots 0}^{11 \ldots 1} \theta_{\alpha} L_{\alpha}=\sum_{\alpha=00 \ldots 0}^{11 \ldots 1} \lambda_{\alpha} R_{\alpha}=\sum_{\alpha=00 \ldots 0}^{11 \ldots 1} \rho_{\alpha} M_{\alpha}$

where $L_{\alpha}, R_{\alpha}$, and $M_{\alpha}$ are given by Definition 1. The $\lambda_{\alpha}$ are the eigenvalues of $V$ and the $\rho_{\alpha}$ are the elements of $V$. Furthermore, if $\lambda, \rho$ and $\theta$ denote the $2^{p} \times 1$ vectors with elements $\lambda_{\alpha}, \rho_{\alpha}$, and $\theta_{\alpha}$, we have $\lambda=K_{0} \theta$ and $\rho=K \theta$, with $K$ and $K_{0}$ given by (11) and (12).

Proof. Equation (4) may also be written as

$V=\left(\theta^{\prime} \otimes I_{N}\right) L$

where

$L=\left(\begin{array}{c}L_{00 \ldots 0} \\ \vdots \\ L_{11 \ldots 1}\end{array}\right)$

and where the matrices $L_{\alpha}$ are as defined in (13). Using $\lambda=K_{0} \theta$ and $\rho=K \theta,(24)$ is equivalent to

$$
\begin{aligned}
V & =\left(\theta^{\prime} \otimes I_{N}\right)\left(K_{0}^{\prime} \otimes I_{N}\right)\left(\left(K_{0}^{\prime}\right)^{-1} \otimes I_{N}\right) L \\
& =\left(\lambda^{\prime} \otimes I_{N}\right)\left(\left(K_{0}^{\prime}\right)^{-1} \otimes I_{N}\right) L \equiv\left(\lambda^{\prime} \otimes I_{N}\right) R
\end{aligned}
$$

and

$$
\begin{aligned}
V & =\left(\theta^{\prime} \otimes I_{N}\right)\left(K^{\prime} \otimes I_{N}\right)\left(\left(K^{\prime}\right)^{-1} \otimes I_{N}\right) L \\
& =\left(\rho^{\prime} \otimes I_{N}\right)\left(\left(K^{\prime}\right)^{-1} \otimes I_{N}\right) L \equiv\left(\rho^{\prime} \otimes I_{N}\right) M .
\end{aligned}
$$

If we partition $R$ and $M$ in the same fashion as $L$,

$R=\left(\begin{array}{c}R_{00 \ldots 0} \\ \vdots \\ R_{11 \ldots 1}\end{array}\right)$ and $M=\left(\begin{array}{c}M_{00 \ldots 0} \\ \vdots \\ M_{11 \ldots 1}\end{array}\right)$,

it follows from (25) and (26) that

$R_{\beta}=\sum_{\alpha=00 \ldots 0}^{11 \ldots 1} k_{\beta \alpha}^{0} L_{\alpha}$ 
and

$M_{\beta}=\sum_{\alpha=00 \ldots 0}^{11 \ldots 1} k_{\beta \alpha} L_{\alpha}$

where $k_{\beta \alpha}^{0}$ and $k_{\beta \alpha}$ are the elements in row $\beta$ and column $\alpha$ of $\left(K_{0}^{\prime}\right)^{-1}$ and $\left(K^{\prime}\right)^{-1}$, respectively.

We now show that (27) is the expansion of (14). When $p=1$, we have

$$
\left(K_{0}^{\prime}\right)^{-1}=n_{p}^{-1}\left(\begin{array}{cc}
1 & 0 \\
-1 & n_{p}
\end{array}\right), \quad L_{0}=S_{n_{p}}, \quad L_{1}=I_{n_{p}} \text {, }
$$

so that (27) obviously implies (14). It is easy to see that the property is true for $p$ whenever it is true for $p-1$. Indeed, $\left(K_{0}^{\prime}\right)^{-1} \equiv\left(K_{0}^{\prime}(p)\right)^{-1}$ and $L \equiv L(p)$ may be defined recursively as

$$
\begin{gathered}
\left(K_{0}^{\prime}(p)\right)^{-1}=\left(\begin{array}{cc}
n_{1}^{-1}\left(K_{0}^{\prime}(p-1)\right)^{-1} & 0 \\
-n_{1}^{-1}\left(K_{0}^{\prime}(p-1)\right)^{-1} & \left(K_{0}^{\prime}(p-1)\right)^{-1}
\end{array}\right) \\
L(p)=\left(\begin{array}{c}
S_{n_{1}} \otimes L_{00 \ldots 0}(p-1) \\
\vdots \\
S_{n_{1}} \otimes L_{11 \ldots 1}(p-1) \\
I_{n_{1}} \otimes L_{00 \ldots 0}(p-1) \\
\vdots \\
I_{n_{1}} \otimes L_{11 \ldots 1}(p-1)
\end{array}\right)
\end{gathered}
$$

so that, upon letting $\beta=\left(\beta_{1} \gamma_{1} \gamma_{2} \cdots \gamma_{p-1}\right)$, (27) may be written if $\beta_{1}=0$ as

$$
\begin{aligned}
R_{\beta}(p) & =\sum_{\alpha}\left(n_{1}^{-1} k_{\gamma \alpha}^{0}(p-1)\right)\left(S_{n_{1}} \otimes L_{\alpha}(p-1)\right) \\
& =n_{1}^{-1} S_{n_{1}} \otimes \sum_{\alpha} k_{\gamma \alpha}^{0}(p-1) L_{\alpha}(p-1) \\
& =n_{1}^{-1} S_{n_{1}} \otimes R_{\gamma}(p-1)
\end{aligned}
$$

and if $\beta_{1}=1$ as:

$$
\begin{aligned}
R_{\beta}(p)= & \sum_{\alpha}\left(-n_{1}^{-1} k_{\gamma \alpha}^{0}(p-1)\right)\left(S_{n_{1}} \otimes L_{\alpha}(p-1)\right) \\
& +\sum_{\alpha}\left(k_{\gamma \alpha}^{0}(p-1)\right)\left(I_{n_{1}} \otimes L_{\alpha}(p-1)\right) \\
= & \left(I_{n_{1}}-n_{1}^{-1} S_{n_{1}}\right) \otimes \sum_{\alpha} k_{\gamma \alpha}^{0}(p-1) L_{\alpha}(p-1) \\
= & \left(I_{n_{1}}-n_{1}^{-1} S_{n_{1}}\right) \otimes R_{\gamma}(p-1)
\end{aligned}
$$

where we note that $\alpha$ and $\gamma$ now involve $p-1$ binary digits rather than $p$. The proof that $(28)$ is the expansion of (15) is exactly similar.

We must show that the matrices $R_{\beta}$ in (14) are idempotent, pairwise orthogonal, and add up to an identity matrix, thus proving our claim that $\lambda$ is indeed the vector 
of eigenvalues. $R_{\beta} R_{\beta}=R_{\beta}$ is obvious since $R_{\beta}$ is a Kronecker product of idempotent matrices. $R_{\alpha} R_{\beta}=O$ for $\alpha \neq \beta$ follows from the fact that $n_{i}^{-1} S_{n_{i}}\left(I_{n_{i}}-n_{i}^{-1} S_{n_{i}}\right)=$ $O$; indeed, $\alpha \neq \beta$ implies the existence of an index $i$ such that $\alpha_{i}=0$ and $\beta_{i}=1$, or $\alpha_{i}=1$ and $\beta_{i}=0$. So $\sum_{\beta} R_{\beta}$ is symmetric and idempotent; in order to show that it equals $I_{N}$, we show that its trace is nonzero. This follows easily from (27), which implies

$\operatorname{tr}\left(\sum_{\beta=00 \ldots 0}^{11 \ldots 1} R_{\beta}\right)=\sum_{\beta=00 \ldots 0}^{11 \ldots 1} \sum_{\alpha=00 \ldots 0}^{11 \ldots 1} k_{\beta \alpha}^{0} \operatorname{tr}\left(L_{\alpha}\right)=N \sum_{\beta=00 \ldots 0}^{11 \ldots 1} \sum_{\alpha=00 \ldots 0}^{11 \ldots 1} k_{\beta \alpha}^{0}=N$

since $\Sigma_{\beta} \Sigma_{\alpha} k_{\beta \alpha}^{0}$ is the sum of the elements of $\left(K_{0}^{\prime}\right)^{-1}$, which is easily seen to equal unity:

$s^{\prime}\left(K_{0}^{\prime}\right)^{-1} s=\left(\bigotimes_{i=1}^{p}\left(\begin{array}{ll}1 & 1\end{array}\right)\right)\left(\bigotimes_{i=1}^{p}\left(\begin{array}{cc}n_{i}^{-1} & 0 \\ -n_{i}^{-1} & 1\end{array}\right)\right)\left(\bigotimes_{i=1}^{p}\left(\begin{array}{l}1 \\ 1\end{array}\right)\right)=1$.

It was shown in Deschamps [4] that the elements of $\rho$ are also the elements of $V$. Since, from (15), the elements of $M_{\beta}$ are 0 or 1 , this is also implied by $\Sigma_{\beta} M_{\beta}=S_{N}$, which is readily verified from (28).

\section{APPENDIX B. PROOF OF THEOREM 1}

It is an immediate consequence of Lemma 1 that $V, V_{*}$, and $V_{*}-V$ can be expressed as (different) linear combinations of the same matrices $R_{\alpha}$. The coefficients in the linear combinations are the eigenvalues; in the case of $V_{*}-V$, they form the vector $K_{0} \theta^{*}-K_{0} \theta=K_{0} \eta$.

As is easily seen from the properties of the matrices $R_{\alpha}$, both $V_{*}^{-1 / 2}$ and $D$ can again be represented as linear combinations of the same matrices $R_{\alpha}$. The eigenvalues of $D$ are obtained by multiplying the eigenvalues of $V_{*}-V$ by those of $V_{*}^{-1}$, so that they form the vector $\Delta^{-1} K_{0} \eta$, with elements $\Sigma_{\gamma} \eta_{\gamma} a_{\beta \gamma}$. This proves that (16) is indeed the spectral form of $D$.

In order to obtain equation (17), we substitute (27) into (16):

$D=\sum_{\gamma=00 \ldots 0}^{11 \ldots 1} \eta_{\gamma} \sum_{\beta=00 \ldots 0}^{11 \ldots 1} a_{\beta \gamma} \sum_{\alpha=00 \ldots 0}^{i 1 \ldots 1} k_{\beta \alpha}^{0} L_{\alpha}=\sum_{\gamma=00 \ldots 0}^{11 \ldots 1} \eta_{\gamma} \sum_{\alpha=00 \ldots 0}^{11 \ldots 1}\left(\sum_{\beta=00 \ldots 0}^{11 \ldots 1} k_{\beta \alpha}^{0} a_{\beta \gamma}\right) L_{\alpha}$ which is equivalent to (17) since $k_{\beta \alpha}^{0}$ is the element in row $\alpha$ and column $\beta$ of $K_{0}^{-1}$.

In order to obtain equation (18), we note that (25) and (26) imply $R=\left(\left(K_{0}^{\prime}\right)^{-1} \otimes\right.$ $\left.I_{N}\right) L$ and $L=\left(K^{\prime} \otimes I_{N}\right) M$, so that

$R=\left(\left(K_{0}^{\prime}\right)^{-1} K^{\prime} \otimes I_{N}\right) M$

and we may write, similarly to (27) and (28),

$R_{\beta}=\sum_{\alpha=00 \ldots 0}^{11 \ldots 1} k_{\beta \alpha}^{*} M_{\alpha}$ 
where $k_{\beta \alpha}^{*}$ is the element in row $\beta$ and column $\alpha$ of $\left(K K_{0}^{-1}\right)^{\prime}$. Upon substituting (29) into (16), we obtain

$D=\sum_{\gamma=00 \ldots 0}^{11 \ldots 1} \eta_{\gamma} \sum_{\beta=00 \ldots 0}^{11 \ldots 1} a_{\beta \gamma} \sum_{\alpha=00 \ldots 0}^{11 \ldots 1} k_{\beta \alpha}^{*} M_{\alpha}=\sum_{\gamma=00 \ldots 0}^{11 \ldots 1} \eta_{\gamma} \sum_{\alpha=00 \ldots 0}^{11 \ldots 1}\left(\sum_{\beta=00 \ldots 0}^{11 \ldots 1} k_{\beta \alpha}^{*} a_{\beta \gamma}\right) M_{\alpha}$ and (18) follows upon noting that $k_{\beta \alpha}^{*}$ is the element in row $\alpha$ and column $\beta$ of $K K_{0}^{-1}$.

\section{APPENDIX C. ESTIMATION OF THE VARIANCE COMPONENTS}

This appendix may be viewed as a generalization of the first part of Amemiya [1] to the $p$-way classification; however, the method of proof is partly new and relies on Lemma 1 . We first note that, since $\theta=K_{0}^{-1} \lambda$, unbiased estimators of the variance components are easy to obtain from unbiased estimators of the eigenvalues; however, since $\theta_{00 \ldots o}=0$ is known and since $K_{0}^{-1}$ is upper triangular, we need only estimate the last $2^{p}-1$ eigenvalues. For this reason we will always assume in this appendix that $\alpha \neq 00 \ldots 0$.

We will first present unbiased estimators $\bar{\lambda}_{\alpha}$ of the eigenvalues when the true disturbances are known. The $2^{p}-1$ last equations of $\tilde{\theta}=K_{0}^{-1} \bar{\lambda}$ then provide unbiased estimators of the variance components $\theta_{\alpha}$.

We will show that $\tilde{\theta}_{\alpha}$ is consistent under the normality of $u$ by proving that $V\left(\tilde{\theta}_{\alpha}\right) \rightarrow 0$; the usual continuity argument does not apply since $K_{0}^{-1}$ is a function of the numbers of observations $n_{i}$. We will then show that predicting the true disturbances by the analysis of covariance residuals (a procedure suggested by Amemiya) leads to estimated variance components $\hat{\theta}_{\alpha}$ with the same asymptotic distribution as the $\bar{\theta}_{\alpha}$. It follows that $\hat{\theta}_{\alpha}$ is a consistent, and asymptotically unbiased, estimator of $\theta_{\alpha}$.

In the omitted component case of Section 3 , we let $\hat{\theta}_{\gamma}^{*}=0$, and $\hat{\theta}_{\alpha}^{*}=\hat{\theta}_{\alpha}$ for $\alpha \neq$ $\gamma$; misspecification does not affect the consistency of $\hat{\theta}_{\alpha}^{*}$ since $\lambda$ is estimated from the correctly specified model.

We first show that when the true disturbances are known, unbiased estimators of the eigenvalues are given by $\bar{\lambda}_{\alpha}=\mu_{\alpha}^{-1} u^{\prime} R_{\alpha} u$, where, from (14), $\mu_{\alpha}=\operatorname{tr}\left(R_{\alpha}\right)=$ $\Pi_{i=1}^{p}\left(n_{i}-1\right)^{\alpha_{i}}$. This follows from Balestra [2, Lemmá 1]:

$E\left(\tilde{\lambda}_{\alpha}\right)=\mu_{\alpha}^{-1} \operatorname{tr}\left(R_{\alpha} V\right)=\mu_{\alpha}^{-1} \operatorname{tr}\left(R_{\alpha} \sum_{\beta} \lambda_{\beta} R_{\beta}\right)=\lambda_{\alpha} \mu_{\alpha}^{-1} \operatorname{tr}\left(R_{\alpha}\right)=\lambda_{\alpha}$.

Under the normality of $u$, we have, from Balestra [2, Lemma 6]:

$V\left(u^{\prime} R_{\alpha} u\right)=2 \operatorname{tr}\left(R_{\alpha} V R_{\alpha} V\right)=2 \operatorname{tr}\left(\lambda_{\alpha}^{2} R_{\alpha}\right)=2 \lambda_{\alpha}^{2} \mu_{\alpha} ;$

furthermore $u^{\prime} R_{\alpha} u$ and $u^{\prime} R_{\beta} u$ are independent for $\alpha \neq \beta$. Upon letting $\bar{\theta}_{\alpha}=\Sigma_{\beta} c_{\alpha \beta} \bar{\lambda}_{\beta}$ with $c_{\alpha \beta}$ the element in row $\alpha$ and column $\beta$ of $K_{0}^{-1}$, we then have

$V\left(\bar{\theta}_{\alpha}\right)=2 \sum_{\beta} c_{\alpha \beta}^{2} \mu_{\beta}^{-1} \lambda_{\beta}^{2}=2 \lambda^{\prime} \Delta \Delta_{\alpha} \lambda=2 \theta^{\prime} K_{0}^{\prime} \Delta \Delta_{\alpha} K_{0} \theta$ 
where $\Delta$ and $\Delta_{\alpha}$ are diagonal matrices with the $\mu_{\beta}^{-1}$ and the $c_{\alpha \beta}^{2}$ on the diagonal, respectively. It may be checked that

$$
\begin{aligned}
& \Delta=\frac{1}{\prod_{i=1}^{p}\left(n_{i}-1\right)} \bigotimes_{i=1}^{p}\left(\begin{array}{cc}
n_{i}-1 & 0 \\
0 & 1
\end{array}\right) \\
& \Delta_{\alpha}=\frac{1}{N^{2}} \bigotimes_{i=1}^{p}\left(\begin{array}{cc}
0 & 0 \\
0 & n_{i}^{2}
\end{array}\right)^{\alpha_{i}}
\end{aligned}
$$

so that the matrix in the quadratic form (30) may be written

$$
\begin{aligned}
K_{0}^{\prime} \Delta \Delta_{\alpha} K_{0} & =\frac{1}{N^{2} \prod_{i=1}^{p}\left(n_{i}-1\right)} \bigotimes_{i=1}^{p}\left(\begin{array}{cc}
n_{i} & 0 \\
1 & 1
\end{array}\right) \bigotimes_{i=1}^{p}\left(\begin{array}{cc}
n_{i}-1 & 0 \\
0 & 1
\end{array}\right) \\
\bigotimes_{i=1}^{p}\left(\begin{array}{cc}
0 & 0 \\
0 & n_{i}^{2}
\end{array}\right)^{\alpha_{i}} \bigotimes_{i=1}^{p}\left(\begin{array}{cc}
n_{i} & 1 \\
0 & 1
\end{array}\right) & \frac{1}{N^{2} \prod_{i=1}^{p}\left(n_{i}-1\right)} \bigotimes_{i=1}^{p}\left(\begin{array}{cc}
0 & 0 \\
0 & n_{i}^{2}
\end{array}\right)^{\alpha_{i}}\left(\begin{array}{cc}
n_{i}^{2}\left(n_{i}-1\right) & n_{i}\left(n_{i}-1\right) \\
n_{i}\left(n_{i}-1\right) & n_{i}
\end{array}\right)^{1-\alpha_{i}} \\
& =\bigotimes_{i=1}^{p}\left(\begin{array}{cc}
0 & 0 \\
0 & \left(n_{i}-1\right)^{-1}
\end{array}\right)^{\alpha_{i}}\left(\begin{array}{cc}
1 & n_{i}^{-1} \\
n_{i}^{-1} & n_{i}^{-1}\left(n_{i}-1\right)^{-1}
\end{array}\right)^{1-\alpha_{i}} \stackrel{\text { def }}{=} P_{\alpha} .
\end{aligned}
$$

It is easily seen from (33) that $\lim P_{\alpha}=O$, so that $V\left(\tilde{\theta}_{\alpha}\right) \rightarrow 0$ in (30). Furthermore, it can be checked that $\lim \mu_{\alpha} P_{\alpha}=e_{\alpha} e_{\alpha}^{\prime}$, where $e_{\alpha}$ is the $2^{p} \times 1$ unit vector with element $\alpha$ equal to unity; equation (30) then implies $\lim \mu_{\alpha} V\left(\tilde{\theta}_{\alpha}\right)=2 \theta_{\alpha}^{2}$. This result, as well as equations (30) and (33), is consistent with Amemiya [1, pp. 5 and 6].

We now show the asymptotic equivalence when $u$ is replaced by $\hat{u}=M y=M u$, where

$M=I_{N}-\frac{1}{N} S_{N}-\left(I_{N}-\frac{1}{N} S_{N}\right)\left(X\left(X^{\prime} Q X\right)^{-1} X^{\prime} Q\right)$

with $Q \equiv R_{11 \ldots 1}=\bigotimes_{i=1}^{p}\left(I_{n_{i}}-n_{i}^{-1} S_{n_{i}}\right.$ ) (see Amemiya [1]). Since $R_{\alpha} S_{N}=S_{N} R_{\alpha}=$ $O$, we have

$M^{\prime} R_{\alpha} M=R_{\alpha}-Q^{\prime} X\left(X^{\prime} Q X\right)^{-1} X^{\prime} R_{\alpha} X\left(X^{\prime} Q X\right)^{-1} X^{\prime} Q$.

Hence, upon letting

$\hat{\theta}_{\alpha}=\sum_{\beta} c_{\alpha \beta} \hat{\lambda}_{\beta}=\sum_{\beta} c_{\alpha \beta} \mu_{\beta}^{-1} \hat{u}^{\prime} R_{\beta} \hat{u}$

we see that ${ }^{4}$

$$
\begin{aligned}
\sqrt{\mu_{\alpha}}\left(\hat{\theta}_{\alpha}-\theta_{\alpha}\right)= & \sqrt{\mu_{\alpha}}\left(\bar{\theta}_{\alpha}-\theta_{\alpha}\right) \\
& -\sqrt{\mu_{\alpha}} \sum_{\beta} c_{\alpha \beta} \mu_{\beta}^{-1} \frac{u^{\prime} Q X}{\sqrt{N}}\left(\frac{X^{\prime} Q X}{N}\right)^{-1}\left(\frac{X^{\prime} R_{\beta} X}{N}\right)\left(\frac{X^{\prime} Q X}{N}\right)^{-1} \frac{X^{\prime} Q u}{\sqrt{N}} .
\end{aligned}
$$


The estimators $\hat{\theta}_{\alpha}$ and $\tilde{\theta}_{\alpha}$ will be asymptotically equivalent if the last terms in (34) converge in probability to zero. If $\left(X^{\prime} Q X\right) / N$ tends to a finite, positive definite matrix and if $\left(X^{\prime} R_{\beta} X\right) / N$ is bounded, this will be true when $\sqrt{\mu_{\alpha}} c_{\alpha \beta} \mu_{\beta}^{-1}$ tends to 0 for all $\beta$, by the same argument as in Amemiya [1, equation (16)]. But $c_{\alpha \beta} \mu_{\beta}^{-1}$ is the element in row $\alpha$ and column $\beta$ of $K_{0}^{-1} \Delta$, where $\Delta$ is defined in (31). Since, from (12) and (31),

$K_{0}^{-1} \Delta=\frac{1}{N \prod_{i=1}^{p}\left(n_{i}-1\right)} \bigotimes_{i=1}^{p}\left(\begin{array}{cc}1 & -1 \\ 0 & n_{i}\end{array}\right)\left(\begin{array}{cc}n_{i}-1 & 0 \\ 0 & 1\end{array}\right)=\frac{1}{N} \bigotimes_{i=1}^{p}\left(\begin{array}{cc}1 & -\left(n_{i}-1\right)^{-1} \\ 0 & n_{i}\left(n_{i}-1\right)^{-1}\end{array}\right)$,

we see that this is indeed the case. 